\title{
Randomised-controlled trial of a web-based dietary intervention for patients with type 2 diabetes mellitus: Study protocol of myDIDeA
}

\author{
Amutha Ramadas ${ }^{1 *}$, Kia Fatt Quek', Carina KY Chan ${ }^{1}$, Brian Oldenburg ${ }^{2}$ and Zanariah Hussein ${ }^{3}$
}

\begin{abstract}
Background: The potential of web-based interventions in dietary behaviour modification of the diabetics has not been fully explored. We describe the protocol of a 12-month match-design randomised controlled trial of a webbased dietary intervention for type 2 diabetic patients with primary aim to evaluate the effect of the intervention on their dietary knowledge, attitude and behaviour (KAB). The secondary objective of this study is to improve the participants' dietary practices, physical measurements and biomarkers.

Methods/Design: A minimum total sample of 82 Type 2 diabetics will be randomised, either to the control group, who will receive the standard diabetes care or the e-intervention group, who will participate in a 6-month webbased dietary intervention in addition to the standard care. The dietary recommendations are based on existing guidelines, but personalised according to the patients' Stages of Change (SOC). The participants will be followed up for 6 months post-intervention with data collection scheduled at baseline, 6-month and 12-month.

Discussion: We are aiming for a net improvement in the KAB score in participants of the e-intervention group, besides investigating the impact of the e-intervention on the dietary practices, physical measurements and blood biomarkers of those patients. The successful outcome of this study can be a precursor for policy makers to initiate more rigorous promotion of such web-based programmes in the country.
\end{abstract}

Trial registration: Clinicaltrials.gov NCT01246687

\section{Background}

Diabetes is a growing concern in Malaysia and in the world. The prevalence of diabetes in adults worldwide was estimated to rise 366 million in the year 2030 from 171 million in 2000 [1]. The prevalence of Type 2 Diabetes Mellitus (T2DM) in Malaysians above 30 years old was reported to be between $11 \%$ and $14 \%$ in 2006 , and it is estimated to rise further [2]. T2DM accounts for a huge burden of morbidity and mortality through micro and macrovascular complications [3,4]. This has lead to an increasing demand on dietary and lifestyle modifications to delay the disease progression.

Web-based interventions have been successfully implemented in improving self-management of diabetes [5-8], physical activity $[9,10]$ and weight management $[11,12]$ in

\footnotetext{
* Correspondence: aram38@monash.student.edu

'Jeffrey Cheah School of Medicine and Health Sciences, Monash University

Sunway Campus, 46150 Bandar Sunway, Petaling Jaya, Malaysia

Full list of author information is available at the end of the article
}

adults with T2DM. A web-based nutrition education for diabetes prevention study among young adults has shown significant reduction in dietary fat intake [13]. To date there is no published study focused solely on dietary behaviour change in adults with T2DM via a website-based system. Nevertheless, dietary modification has been incorporated as a component of multifactorial behavioural interventions in diabetes prevention and management $[11,12,14]$.

The success of a web-based intervention relies heavily on the website log-in rates, usability, and personalisation. Content development in simplified local language improves the usability of the website [15], while regular reinforcement using reminder services such as e-mails and SMS increases the log-in rates [16]. The use of behavioural theories could assist the personalisation of web-based interventions. Transtheoretical Model (TTM) [17] is one of such behavioural model that has been recently used in web-based interventions, especially

\section{Biomed Central}


those focusing on improving dietary and lifestyle behaviours [10,18].

The study protocol for a randomised controlled trial (RCT) of a web-based dietary intervention for patients with T2DM in Malaysia is presented here. We hypothesised that the e-intervention group will show a greater improvement in dietary KAB than the control group. Secondly, we hypothesised that the web-based intervention to results in a better dietary practices, anthropometric measurements and blood biomarkers in the eintervention group than the controls.

\section{Methods/Design}

\section{Study design}

This is a two-armed matched-design randomized controlled trial (RCT) scheduled for 12 months (Figure 1). The study is designed according to the recommendations of the CONSORT statement for randomised trials of non-pharmacologic treatment [19].

The conceptual framework of the study is presented in Figure 2. Twelve dietary modules in the intervention package is personalised according to the patients' Stages of Change (SOC), and is expected to improve their dietary $\mathrm{KAB}$ and assist them to progress in their respective SOC. The improvements in KAB and progress in SOC are expected to be reflected in the patients' dietary practices. Adoption of healthy dietary practices then is expected to be reflected on the anthropometric measurements and biomarkers.

\section{Study aims}

The primary aim of our study is to evaluate the effect of a six month dietary e-intervention on dietary knowledge, attitude and behaviour (KAB) in patients with T2DM.

The secondary aim is to determine the impact of the intervention on dietary practices (nutrient intake, dietary GI, food frequency score, supplements intake, cooking method and eating out habit), physical measurements (height, weight, body mass index, waist circumference, body fat percentage and blood pressure) and blood biomarkers (fasting blood glucose (FBG), glycosylated haemoglobin (HbA1c) and lipid profile).

\section{Ethics approval}

The study has received ethics approval from Malaysian Ethics Research (NMRR-09-303-3416) and Monash University Human Research Ethics Committee (CF09/1583 - 2009000877).

\section{Study sample \\ Sample size and power calculation}

The sample size calculation is based on a difference in fat and fibre-related behaviour score as reported by a previous computer assisted dietary intervention study in type 2 diabetics [20]. Using a reduction in the mean behaviour score from $1.93 \pm 0.5$ (baseline) to $1.69 \pm 0.4$ (post intervention), the GPower software [21] calculated that 31 patients are needed in each group to detect this difference with a two-sided alpha of 0.05 , a power of $80 \%$. Based on $30 \%$ attrition rate for one year, a minimum of 41 participants are required in each group.

\section{Recruitment process}

The eligibility screening, recruitment of study participants and data collections are conducted in the outpatient medical clinic of three urban hospitals in Malaysia. Diabetic nurses provide assistance in identifying potential study participants according to the eligibility criteria (Table 1). If the patients are found have potential to participate in the study, they complete the baseline questionnaire after giving a written informed consent. They are, however, excluded if the baseline dietary KAB score was more than $50 \%$.

\section{Randomisation and treatment allocation}

Eligible diabetic patients who have consented to participate are matched for age, sex and ethnicity, and randomised to either e-intervention or control group.

\section{Control group}

This group receives the usual standard treatment (diabetic control and management) given to patients with T2DM. Although the controls have access to the Internet, they receive neither website log-in information, nor any reinforcement via e-mail or SMS.

\section{e-Intervention group}

This group receives an intensive dietary intervention through the study website, personalised according to the participants' SOC, in addition to the usual standard treatment at the outpatient clinics.

\section{Intervention programme}

\section{Dietary module}

Twelve dietary lesson plans are developed for the intervention based on the Nutrition Recommendations and Interventions for Diabetes by American Diabetes Association [22], Malaysian Clinical Practice Guidelines for Type 2 Diabetes Mellitus [23] and Malaysian Medical Nutrition Therapy Guidelines for Type 2 Diabetes [24]. The intervention's structure and materials are developed using TTM's Stages of Change construct [17] (Table 2). The content of each lesson plan is studied for its relevance to the local community and fine-tuned to suit local context.

\section{Website design}

Suitable features to be highlighted in the website are discussed by a research panel comprising of nutritionist, behavioural psychologists, endocrinologist, public health expert, and a web master. Figure 3 outlines the structure 


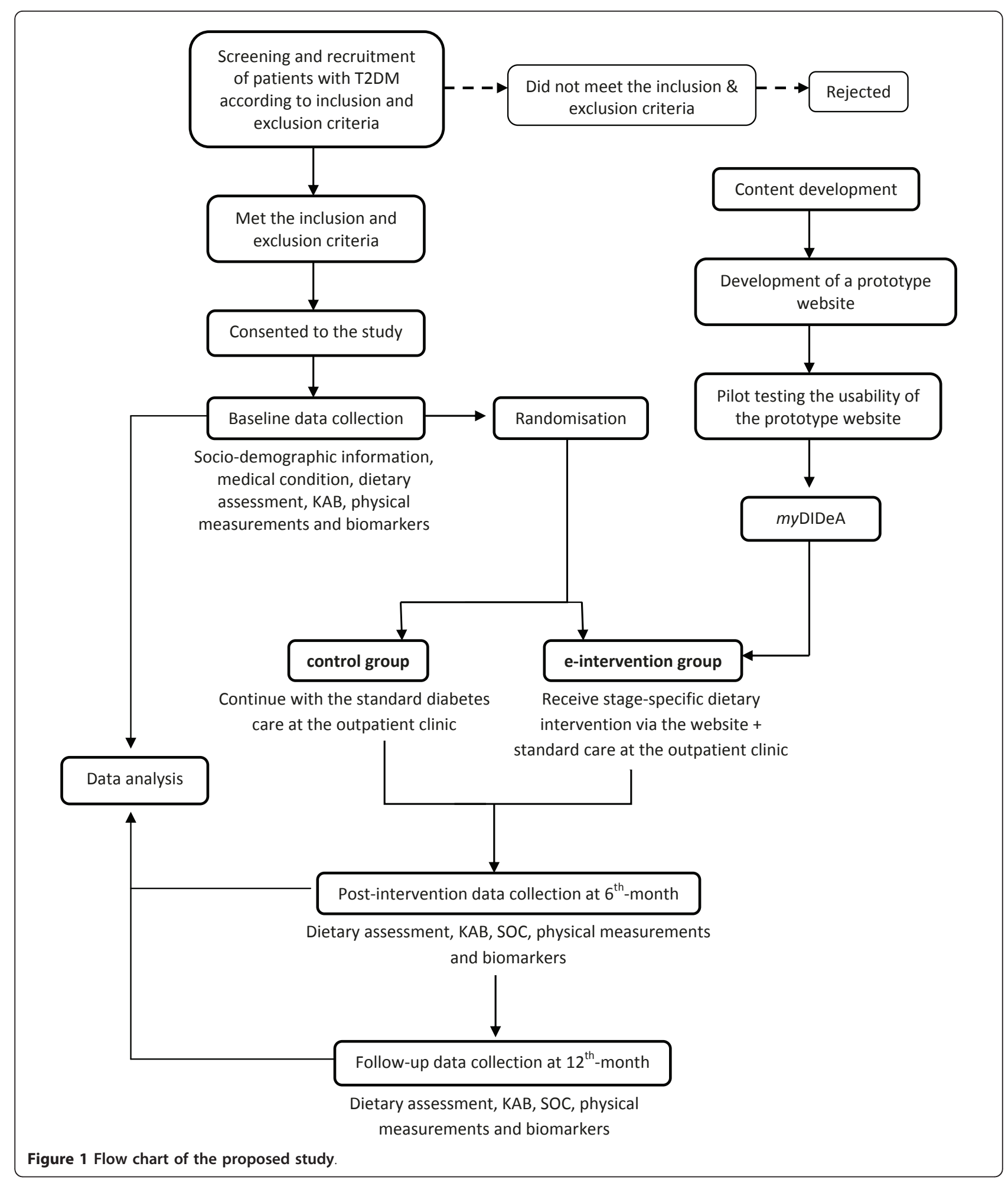

of the study website, myDIDeA (Dietary Intervention for Type 2 Diabetes Patients: An e-Approach).

myDIDeA will be tailored according to the participants' SOC. Each lesson plan has five Likert scale items (strongly agree $=5$ to strongly disagree $=1$ ) and participants are assigned to recommendations based on the score obtained. The recommendations are aimed to address the barriers and motivate the participants according to the lesson plan and stage. Relevant photographs and illustrations are added to enhance the understandability of the lesson 


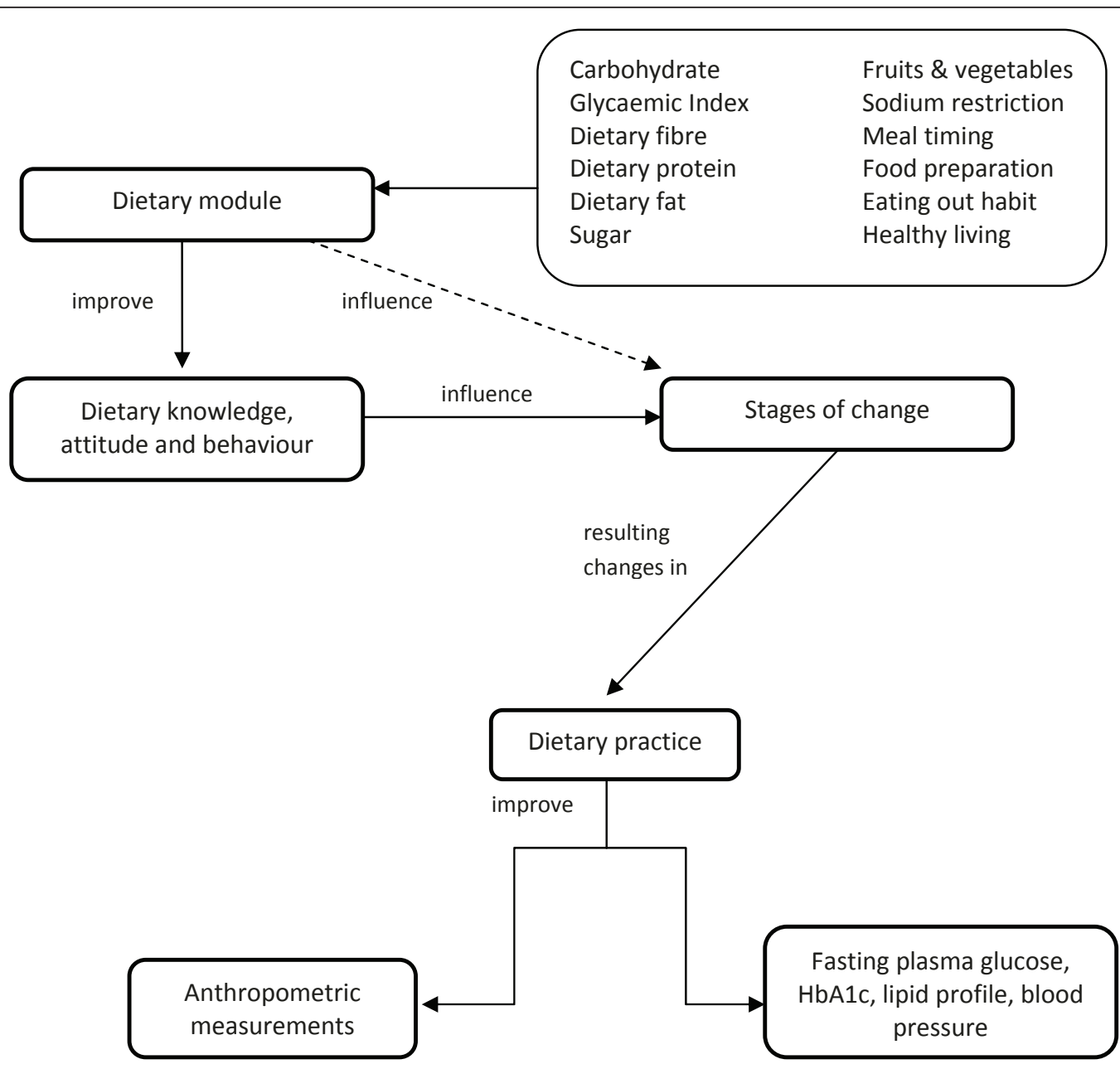

Figure 2 Conceptual framework.

\section{Table 1 Eligibility criteria}

\begin{tabular}{|c|c|}
\hline Inclusion & Exclusion \\
\hline $\begin{array}{l}\text { Mentally sound men and women } \\
\text { who are } \geq 18 \text { years old. } \\
\text { Literate with a fair command of } \\
\text { English and/or Malay languages. } \\
\text { Have access to the Internet at } \\
\text { home, work or public place. } \\
\text { Willing to access the study } \\
\text { website at least once every } \\
\text { fortnight. } \\
\text { Have been confirmed of having } \\
\text { HbA1c of } \geq 7.0 \% \text {. }\end{array}$ & $\begin{array}{l}\text { Pregnant, lactating or intend to } \\
\text { become pregnant during the study } \\
\text { period. } \\
\text { Diagnosed with Type } 1 \text { Diabetes } \\
\text { Mellitus (T1DM) or Gestational } \\
\text { Diabetes Mellitus (GDM) } \\
\text { Weighing more than 150\% of the } \\
\text { desired weight for height. } \\
\text { Any pre-existing condition } \\
\text { compromising the quality of life or } \\
\text { ability to participate according to } \\
\text { protocol. } \\
\text { Have severe complications (chronic } \\
\text { heart disease, cerebrovascular } \\
\text { disease, diagnosed HIV/AIDS, cancer, } \\
\text { emphysema, chronic liver or kidney } \\
\text { disease) that would affect the } \\
\text { subjects' ability to follow the tailored } \\
\text { advice. } \\
\text { Enrolled in other clinical studies. } \\
\text { Having dietary KAB score more than } \\
50 \% \text { at baseline. }\end{array}$ \\
\hline
\end{tabular}

plans. Factsheets and statistics on diabetes, links to existing diabetes resources and an example of ideal health profile also are included.

The participants are briefed on $m y$ DIDeA, and are provided with unique username and password via e-mail or SMS after the randomisation. Log-in reminders are sent to their e-mail each time myDIDeA is updated with new lesson plan. The participants are also encouraged to send their queries to the study nutritionist via the website.

\section{Outcome assessments}

All assessments (Table 3) are conducted at the clinical settings by trained enumerators.

\section{Primary outcomes}

The primary outcome is assessed using a validated interviewer-administered dietary KAB questionnaire in English and Bahasa Malaysia.

\section{Secondary outcomes}

A semi-food frequency questionnaire (SFFQ) is used to record the subjects' dietary intake, while two days 24- 
Table 2 Five Stages of Change, its characteristics and relevant strategies (Kendra C 2010)

\begin{tabular}{|c|c|c|}
\hline Stage & Characteristics & Strategies \\
\hline $\begin{array}{l}\text { Pre- } \\
\text { contemplation }\end{array}$ & $\begin{array}{l}\text { - Not intending to change in the next } 6 \text { months. } \\
\text { - In denial or ignorant of the problem. }\end{array}$ & $\begin{array}{l}\text {-Encourage the patient to self-analyse and rethink his/her } \\
\text { behaviour. } \\
\text {-Explain the risks of current behaviour. }\end{array}$ \\
\hline Contemplation & $\begin{array}{l}\text {-Intending to change in the next } 6 \text { months. } \\
\text {-Having conflicting emotion. }\end{array}$ & $\begin{array}{l}\text {-Weigh the pros and cons of changing behaviour. } \\
\text {-Address barriers and encourage confidence. }\end{array}$ \\
\hline Preparation & $\begin{array}{l}\text {-Planning to change in the next } 30 \text { days and have made a } \\
\text { previous attempt to improve. } \\
\text { - Experimenting and collecting information about change. }\end{array}$ & $\begin{array}{l}\text {-Prepare an action plan or goal. } \\
\text { - Motivate the patient to change. }\end{array}$ \\
\hline Action & $\begin{array}{l}\text {-Taking actions towards achieving the goal for at least the last } \\
6 \text { months. }\end{array}$ & $\begin{array}{l}\text {-Encourage the patient to seek social support and motivate him/ } \\
\text { her to sustain the behaviour. } \\
\text { - Reward for the success. }\end{array}$ \\
\hline Maintenance & - New behaviour is sustained for at least 6 months. & $\begin{array}{l}\text {-Strategies to cope with temptation. } \\
\text { - Reward for the success. }\end{array}$ \\
\hline
\end{tabular}

hour dietary recall is used to analyse the nutrient intake [25]. Besides SFFQ and 24-hour dietary recall, consumption of supplements, cooking techniques and eating out habit for the past one month are also recorded. Anthropometric measurements (body mass index, waist circumference and percentage of body fat) and blood pressure are taken during the interview sessions. The results of participants' blood biomarkers are obtained from their medical record.

\section{Process evaluation}

Adherence to the intervention is assessed by the number of log-ins and duration spent in the website. Besides, the participants' satisfaction of the intervention is assessed by self-administered questionnaire at post-intervention.

\section{Other measurements}

The socio-demographic characteristics, medical history, and smoking and drinking habits are recorded in a structured questionnaire. International Physical Activity Questionnaire (IPAQ) is used to determine the level of physical activity of the participants [26].

\section{Blinding}

It is not possible to blind study nutritionist, webmaster and the participants. The enumerators trained to collect the data, however, are blinded during the data collection.

\section{Statistical analysis}

Axxya Systems Nutritionist Pro ${ }^{\mathrm{TM}}$ Diet Analysis is used to analyze the nutrient intakes and all statistical analyses are performed with IBM $^{\circledR}$ SPSS $^{\circledR}$ Statistics 17.0. Independent $t$-test or equivalent is used to determine differences between the study groups for continuous variables, while $\chi^{2}$ or equivalent is used to determine the association between categorical variables. As this is a prospective RCT involving repeated measures, the ANOVA repeated measures model is applied to observe significant differences within the study groups. The evaluation of the intervention is based on an intention-to- treat analysis, with the $p$ value 0.05 was taken as the level of significance.

\section{Discussion}

This is a 12 months web-based dietary intervention RCT for patients with uncontrolled T2DM with the aim to improve diabetes-related dietary KAB. We are employing existing guidelines in the development of the intervention package that will be modified to suit local context. TTM's SOC construct will be incorporated in the development of the study website, myDIDeA. We are aiming for a net improvement in the KAB score in participants of the eintervention group, besides investigating the impact of the e-intervention on the dietary practices, physical measurements and blood biomarkers of those patients.

We hope to capture the 'teachable moment' to promote dietary behaviour change in diabetics with uncontrolled HbA1c $(\geq 7.0 \%)$ and lower level of diabetes-specific dietary $\mathrm{KAB}(\leq 50 \%$ of total score). We are anticipating an increase in dietary $\mathrm{KAB}$ at the end of the trial, which may contribute to a better blood glucose control and ultimately prevent complication due to diabetes.

Besides offering a theory and evidence based education program, this trial will be utilising bilingual educational materials that have been modified to suit the local content. Culturally sensitive dietary intervention [27] and web content [15] leave greater impact, giving this trial an edge in helping the patients to improve their dietary KAB and achieve better glycaemic control.

This trial is capable of generating a personalised dietary intervention program for a large group of patients. At the same time, the intervention program could be accessed 24 hours using any device connected to the Internet. This will empower the patients to have total control of the intervention materials.

We will be conducting this RCT in urban hospitals, which means the respondents may be of a higher socioeconomic background than average Malaysians. This may 


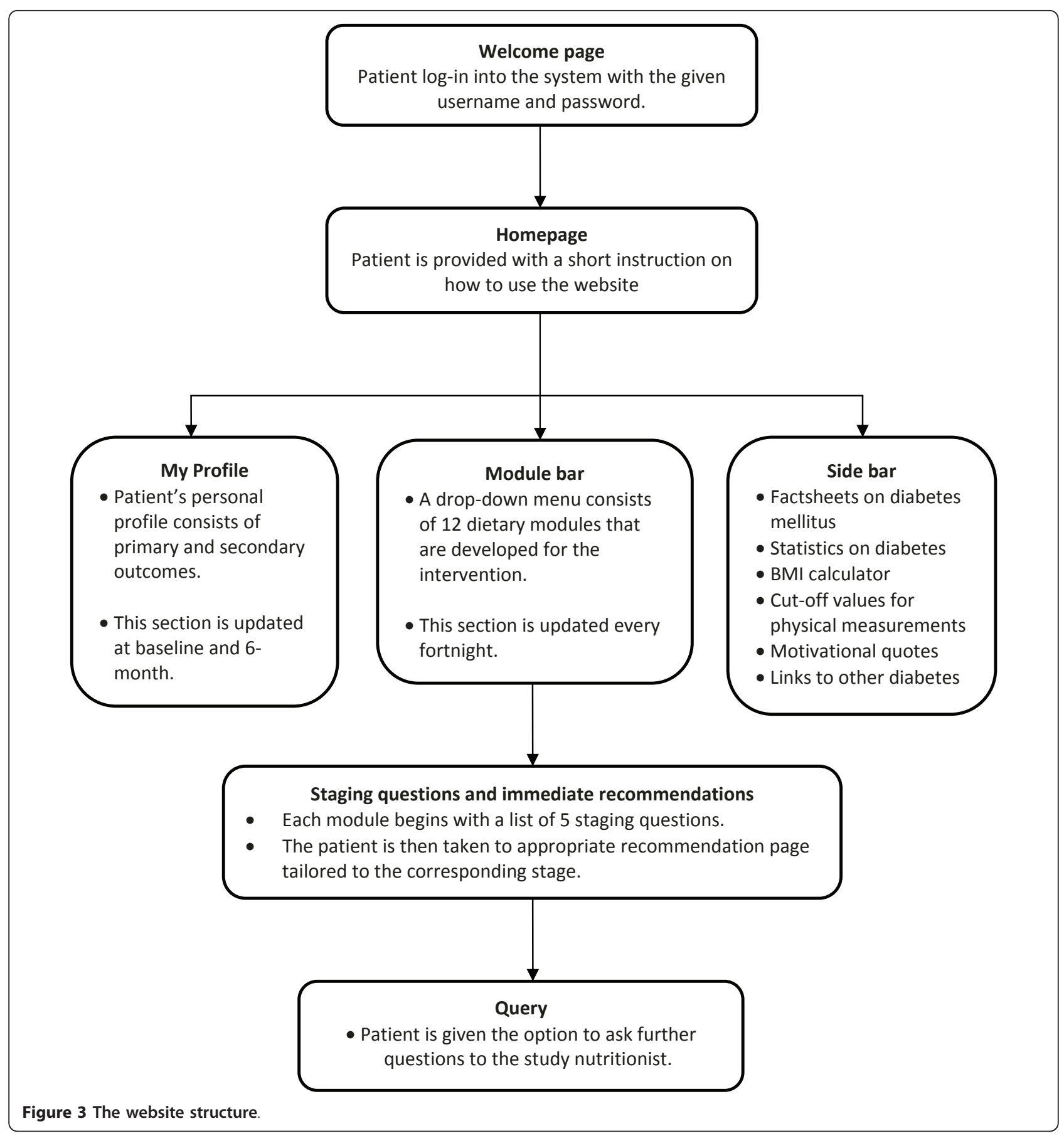

not be representative of the entire population as uptake of internet is different in the urban as compared to the rural. However, we are expecting for a better response to a web-based program in the urban area and it will be interesting to investigate responses from those with higher socio-economic status to an interactive website. This trial can be a model that is first implemented at the urban area for us to evaluate its success. If successful, it can be a precursor for policy makers to initiate more rigorous promotion of such web-based programs to other parts of the country.

Like all web-based interventions, this trial's reach also depends on the participants' responsiveness. Besides personalisation according to SOC, culture and language, the success of this intervention relies on the log-in rates and usability of $m y$ DIDeA. In order to maximise the participation of the trial patients, we will be regularly reinforcing them to log-in via e-mail and SMS. 
Table 3 Assesments that will be carried out in the study

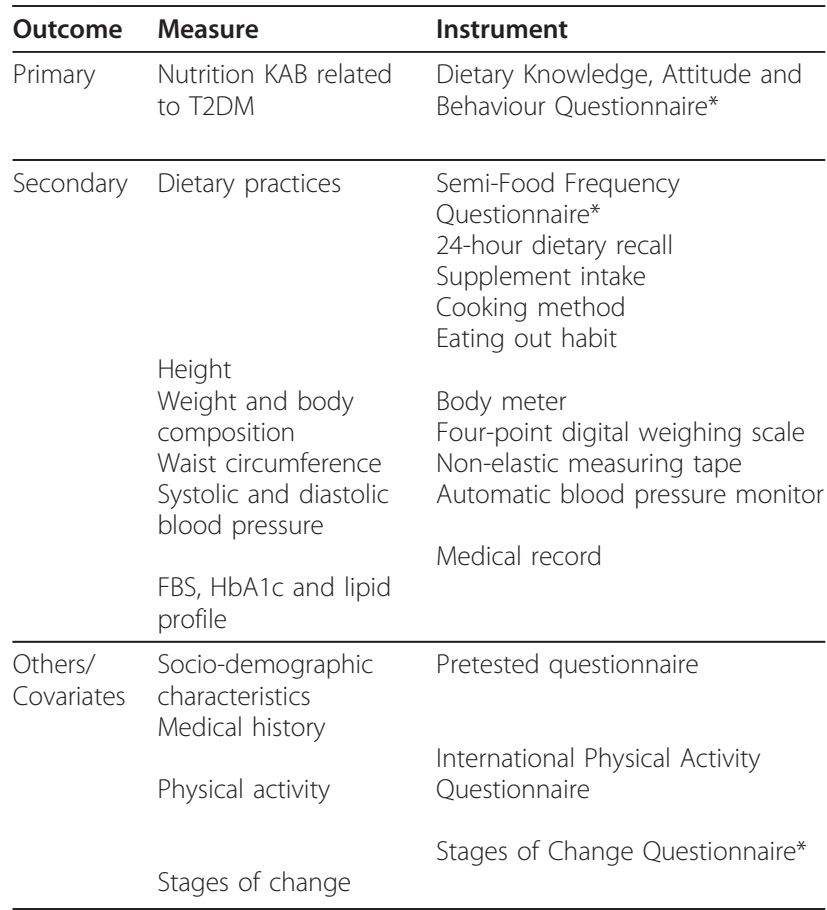

*validated instrument.

Although qualitative assessments such as in-depth interview or focus group discussion were not part of this RCT, the outcome evaluation is supplemented by a detailed process evaluation. The process evaluation is measuring program reach, dose delivered, dose received, fidelity and implementation as well as the participants" satisfaction with the program and acceptability. Collectively, these measures will enable us to draw conclusions about potential program enhancers and barriers if the program were to be delivered in other settings and contexts.

\section{Acknowledgements}

This study is funded by Monash University Sunway Campus (MED2009-CM (SG)-004-QKF),

\footnotetext{
Author details

${ }^{1}$ Jeffrey Cheah School of Medicine and Health Sciences, Monash University Sunway Campus, 46150 Bandar Sunway, Petaling Jaya, Malaysia.

${ }^{2}$ Department of Epidemiology and Preventive Medicine, Monash University Clayton Campus, Wellington Road, Clayton, Victoria 3800, Australia.

${ }^{3}$ Department of Medicine, Hospital Putrajaya, Federal Government Administration Centre, Precinct 7, 62250 Putrajaya, Malaysia.
}

\section{Authors' contributions}

AR developed the study framework and will be involved in data collection and in the development and implementation of the intervention. QKF gave feedback on the research design and will be giving his expertise in data analysis and interpretation of the study results. CKYC and BO gave feedback on the study protocol and to provide expertise in the development of the intervention package. $\mathrm{ZH}$ is to provide expertise in selection of the study sample. All authors read and contributed to the final manuscript.

\section{Competing interests}

The authors declare that they have no competing interests.

Received: 22 February 2011 Accepted: 21 May 2011

Published: 21 May 2011

\section{References}

1. Wild S, Roglic G, Green A, Sicree R, King H: Global prevalence of diabetes: estimates for the year 2000 and projections for 2030. Diabetes Care 2004, 27(5):1047-53.

2. National Health Morbidity Survery III: The Third National Health and Morbidity Survey (NHMS III) 2006. Institute for Public Health, Kuala Lumpur; 2008.

3. Stratton IM, Adler Al, Neil HAW, Matthews DR, Manley SE, Cull CA, Hadden D, Turner RC, Holman RR: Association of glycaemia with macrovascular and microvascular complications of type 2 diabetes (UKPDS 35): prospective observational study. BMJ 2000, 321(7258):405-12.

4. Deshpande AD, Harris-Hayes M, Schootman M: Epidemiology of Diabetes and Diabetes-Related Complications. Phys Ther 2008, 88(11):1254-64.

5. Glasgow RE, Boles SM, McKay HG, Feil EG, Barrera M Jr: The D-Net diabetes self-management program: Long-term implementation, outcomes, and generalization results. Prev Med 2003, 36(4):410-9.

6. Meigs JB, Cagliero E, Dubey A, Murphy-Sheehy P, Gildesgame C, Chueh H, Barry MJ, Singer DE, Nathan DM: A Controlled Trial of Web-Based Diabetes Disease Management: The MGH Diabetes Primary Care Improvement Project. Diabetes Care 2003, 26(3):750-7.

7. Kim HS, Yoo YS, Shim H-S: Effects of an Internet-based Intervention on Plasma Glucose Levels in Patients With Type 2 Diabetes. J Nurs Care Qual 2005, 20(4):335-40

8. Lee TI, Yeh YT, Liu CT, Chen P-L: Development and evaluation of a patient-oriented education system for diabetes management. Int J Med Inform 2007, 76(9):655-63.

9. McKay HG, King D, Eakin EG, Seeley JR, Glasgow RE: The diabetes network internet-based physical activity intervention: a randomized pilot study. Diabetes Care 2001, 24(8):1328-34

10. Kim CJ, Kang DH: Utility of a Web-based Intervention for Individuals With Type 2 Diabetes: The Impact on Physical Activity Levels and Glycemic Control. CIN 2006, 24(6):337-45.

11. Tate DF, Jackvony EH, Wing RR: Effects of Internet Behavioral Counseling on Weight Loss in Adults at Risk for Type 2 Diabetes: A Randomized Trial. JAMA 2003, 289(14):1833-6.

12. McCoy MR, Couch D, Duncan ND, Lynch GS: Evaluating an Internet weight loss program for diabetes prevention. Health Promot Int 2005, 20(3):221-8.

13. Long JD, Armstrong ML, Amos E, Shriver B, Roman-Shriver C, Feng D, Harrison L, Luker S, Nash A, Blevins MW: Pilot Using World Wide Web to Prevent Diabetes in Adolescents. Clin Nurs Res 2006, 15(1):67-79.

14. Wolf AM, Conaway MR, Crowther JQ, Hazen KY, L.Nadler J, Oneida B, Bovbjerg VE: Translating Lifestyle Intervention to Practice in Obese Patients With Type 2 Diabetes. Diabetes Care 2004, 27(7):1570-6.

15. Singh PM, Wight CA, Sercinoglu O, Wilson DC, Boytsov A, Raizada MN: Language Preferences on Websites and in Google Searches for Human Health and Food Information. J Med Internet Res 2007, 9(2):e18.

16. Woodall WG, Buller DB, Saba L, Zimmerman D, Waters E, Hines JM, Cutter GR, Starling R: Effect of emailed messages on return use of a nutrition education website and subsequent changes in dietary behavior. J Med Internet Res 2007, 9(3):e27.

17. Prochaska JO, DiClemente CC: Stages and processes of self-change of smoking: toward an integrative model of change. J Consult Clin Psychol 1983, 51(3):390-5.

18. de Vet $E, d N$ J, de Vries NK, Brug J: The Transtheoretical model for fruit, vegetable and fish consumption: associations between intakes, stages of change and stage transition determinants. Int J Behav Nutr Phys Act 2006, 3:13.

19. Boutron I, Moher D, Altman DG, Schulz KF, Ravaud P, Group ftC: Extending the CONSORT Statement to Randomized Trials of Nonpharmacologic Treatment: Explanation and Elaboration. Ann Intern Med 2008, 148(4):295-309.

20. Glasgow RE, Toobert DJ, Hampson SE, Strycker LA: Implementation, generalization and long-term results of the "choosing well" diabetes self-management intervention. Patient Educ Counsel 2003, 48(2):115-22. 
21. Faul F, Erdfelder E, Lang AG, Buchner A: G*Power 3: A flexible statistical power analysis for the social, behavioral, and biomedical sciences. Behav Res Method 2007, 39:175-91.

22. American Diabetes Association: Nutrition Recommendations and Interventions for Diabetes: A position statement of the American Diabetes Association. Diabetes Care 2008, 31(Supp 1):S61-78.

23. Ministry of Health and Academy of Medicine of Malaysia: Malaysian Clinical Practice Guidelines: Management of Type 2 Diabetes Mellitus., 42009 [http://www.acadmed.org.my/view_file.cfm?fileid=292].

24. Malaysian Dietitians' Association, Ministry of Health: Malaysian Medical Nutrition Therapy for Type 2 Diabetes. Malaysian Dietitians' Association; 2005.

25. Gibson R: Measuring food consumption of individuals.Edited by: Gibson R. Oxford University Press, New York; 2005:

26. Craig CL, Marshall AL, Sjöström M, Bauman AE, Booth ML, Ainsworth BE, Pratt M, Ekelund U, Yngve A, Sallies JF, Oja P: International Physical Activity Questionnaire: 12-Country Reliability and Validity. Med Sci Sports Exerc 2003, 35(8):1381-95

27. Williams JH, Auslander WF, de Groot M, Robinson AD, Houston C, HaireJoshu D: Cultural Relevancy of a Diabetes Prevention Nutrition Program for African American Women. Health Promot Pract 2006, 7(1):56-67.

\section{Pre-publication history}

The pre-publication history for this paper can be accessed here: http://www.biomedcentral.com/1471-2458/11/359/prepub

doi:10.1186/1471-2458-11-359

Cite this article as: Ramadas et al.: Randomised-controlled trial of a web-based dietary intervention for patients with type 2 diabetes mellitus: Study protocol of myDIDeA. BMC Public Health 2011 11:359.

\section{Submit your next manuscript to BioMed Central} and take full advantage of:

- Convenient online submission

- Thorough peer review

- No space constraints or color figure charges

- Immediate publication on acceptance

- Inclusion in PubMed, CAS, Scopus and Google Scholar

- Research which is freely available for redistribution

Submit your manuscript at www.biomedcentral.com/submit 